\title{
Ageing in forensic psychiatric secure settings: The voice of older patients
}

\author{
Claudio Di Lorito, Tom Dening, and Birgit Völlm \\ Division of Psychiatry and Applied Psychology, School of Medicine \\ University of Nottingham
}

Corresponding author: Claudio Di Lorito

Room A10, Institute of Mental Health

University of Nottingham

Triumph Road

Nottingham NG7 2TU

Email: claudio.dilorito@nottingham.ac.uk

\begin{abstract}
Older patients account for around $20 \%$ of the population in secure forensic psychiatric services in the UK. However, little qualitative research has investigated the experience of ageing in secure settings. This study aimed to gather the individual views of a sample of patients over 50 years old in the UK at different levels of security. Fifteen participants were selected and underwent one-on-one qualitative interviews. The interviews were analysed through thematic analysis, which generated seven themes: Self-agency, activities, social life, practical matters, recovery, physical health and service improvement. Study findings highlighted the complexity of ageing in secure settings. Despite the positive feedback reported in aspects such as physical health care, education opportunities, staff and support of religious practices, participants experienced added barriers to recovery, caused by social isolation/withdrawal and activities/treatment that did not respond to their complex age-related needs, generating poor motivation to engage. Our findings call for the development/implementation of programmes tailored to the unique needs of older patients. This process requires an active involvement of the primary stakeholders and further patientcentred research.
\end{abstract}

Keywords: Older patients, forensic psychiatry, old age psychiatry, secure services, qualitative research

Disclaimer: This research was funded by the National Institute for Health Research (NIHR) Collaboration for Leadership in Applied Health Research and Care East Midlands (CLAHRC 
EM). The views expressed in this article are those of the author(s) and not necessarily those of the NHS, the NIHR or the Department of Health and Social Care.

Conflict of interest: None to declare

Number of words: 5,121 


\section{Introduction}

Secure forensic mental health services in England and Wales offer specialist/intensive treatment to mentally ill individuals who, given their psychiatric symptoms, may pose a risk of harm to themselves or others.

In the last decade, the number of older patients in UK secure forensic psychiatric settings has increased. In 1995 , Wong et al. reported that $8 \%$ of the total patients' population in Broadmoor Hospital, a high secure hospital in the UK, was over 50 years old. In 2018, the over 50 s constitute $21 \%$ of the patients in Rampton Hospital, another high secure service (Di Lorito, Dening, \& Völlm, 2018).

Demographic trends (e.g. longer life-expectancy), more frequent prosecution of historic offences, particularly sexual offences and changes in the legal system (e.g. increased number of lifetime sentences) have caused an accumulation of long-stay and newly admitted older patients in forensic psychiatric care (RECOOP, 2015; Yorston, 2015; Moll, 2013; Frazer, 2003).

The few existing studies on older patients found that they experience complex care needs and long-term institutionalisation which may potentially hinder their recovery (Lightbody, Gow, \& Gibb, 2010; Yorston \& Taylor, 2009; Shah, 2006; Tomar, Treasden, \& Shah, 2005; Curtice, Parker, Wismayer, \& Tomison, 2003; Coid, Fazel \& Khatan, 2002; Paradis, Broner, Maher, \& O'Rourke, 2000). However, this evidence is almost entirely based on staff reports and clinical data, which neglects the views of the patients. We therefore deemed it timely to undertake this patient-centred research.

We were driven in our inquiry by: (i). The NICE recommendations on mental wellbeing and independence in older people (2015), which advocates the involvement of older people, the 'expert', in research; (ii). The Recovery Model of forensic psychiatric care (Drennan et al., 
2014), which places strong emphasis on human agency (Ward, Mann \& Gannon, 2007), thus implying an imperative for patient-based research.

Our study aimed to answer the following questions: (i). What is the experience of older patients in forensic psychiatric services? (ii). Are these services meeting their needs? (iii). What can be done to enhance their experience and recovery opportunities?

\section{Methods}

This study was part of a larger project on older patients in three forensic psychiatric settings (high, medium and low secure), within Nottinghamshire Healthcare NHS Foundation Trust. The project comprised a quantitative element (Di Lorito, Dening, \& Völlm, 2018) and a qualitative study around patients' experience (presented here).

\section{Participants}

Inclusion criteria:

1. aged 50 years old or above, in line with current conventions in old age forensic research (Cooney and Braggins, 2010; Baldwin and Leete, 2012; Moll, 2013).

2. been resident in the service for at least one year, to have experience of secure settings.

3. able to give full consent, determined by the researcher.

4. no reasons for not participating identified by the clinical team.

Study participants were selected from the larger project sample through purposive sampling, to ensure that they were representative of the population over 50 in the services and that they could provide rich narratives about their experience. 


\section{Data collection}

Study participants were interviewed individually using a semi-structured interview schedule, but new topics that emerged during the session were also explored to ensure that all relevant information was captured. The interview schedule comprised 25 questions including: (i) Likert-type opening prompts to stimulate participants' reflection and administered orally through an easy-read response sheet; (ii) open-ended questions for further in-depth investigation. It was informed by results from the following pre-study groundwork undertaken by the research team:

(i) Systematic reviews and empirical studies around older forensic populations (Di Lorito, Dening, \& Völlm, 2018; Di Lorito, Völlm, \& Dening, 2018; Di Lorito, Völlm, \& Dening, 2017; Di Lorito, Castelletti, Tripi, Gandellini, Dening, \& Völlm, 2017).

(ii) Review of the NICE guidelines on mental wellbeing and independence in older people (NICE, 2015).

(iii) The primary needs that forensic services should address to promote patients' recovery, as indicated in the Good Lives Model for offender rehabilitation (https://www.goodlivesmodel.com/information).

(iv) One Patient and Public Involvement (PPI) exercise carried out with three patients in Rampton Hospital. Through this exercise, we gathered feedback around the interview questions, and edited the schedule to make it more relevant to the patients.

Interviews were audio-recorded and transcribed by the lead researcher. Data were collected until saturation was reached. 


\section{Data analysis}

The interviews were transcribed, transferred onto NVivo software Version 11 (QSR International Pty Ltd., 2012) and analysed through thematic analysis, a method for identifying, analysing and reporting themes within data (Braun \& Clarke, 2006). We adopted a semi-deductive approach to thematic analysis, and set some initial themes based on our preliminary groundwork. We examined the transcripts and assessed the fitness of these themes.

Where data did not fit into the existing themes, we would generate a tentative new theme. All themes were expanded, restricted, or merged through a back-and-forth process, until a final code book (Table 1) was agreed upon by the whole research team. Relevant data were then extracted and categorised into the final themes.

\section{Ethical approval and data protection}

Ethics approval (REC reference: 16/EM/0505) was obtained by the Health Research Authority (HRA) and permission to run the study by Nottinghamshire Healthcare NHS Foundation Trust Research \& Development (R\&D). We abided by the Declaration of Helsinki (World Medical Association, 2013), Good Clinical Practice (GCP), the Department of Health Research Governance Framework for Health and Social Care (Department of Health, 2005) and the Data Protection Act 1998. To safeguard the patients' anonymity, we did not label any quotation included in this paper (e.g. gender, age of participant).

\section{Results}

We interviewed six patients from a high secure setting, seven from a medium secure setting and two from a low secure setting. Participants were from the male mental health $(n=7)$, personality disorder $(n=6)$, and female services $(n=2)$. Interview administration time averaged 39 minutes (Range: 18-57 minutes). 
The initial list of themes (based on our preliminary groundwork) included: Social life, recovery, self-agency, activities and service improvement. The new themes, which emerged from the participants' interviews were: Practical matters and physical health. We derived seven final themes, each of which included several sub-themes (Table 1). These are discussed below.

\section{Self-agency}

The theme of self-agency explored how much in control patients felt in shaping their own present and future. Most patients felt they were in control of some specific practical aspects of daily living:

'I have control on the little things. I bought myself a couple of dresses, and put on some make up, just things to put me up a bit'

Others felt that they had decision-making power to shape their own treatment:

'The OT staff come on the ward and say, 'We would like you to go on this group'. I think about it and make a choice'

Participants also acknowledged that relinquishing a degree of control was to be expected within secure units:

'We have to realise that we are in a secure unit and there are parts of our lives we can't change'

However, several patients, perceived that control was totally in the hands of members of staff:

I haven't been in control of my own life since I've been locked away. I've had people telling me when I need to get up, what time to eat and what I can't do' 
More often, the temporary loss of decision power constituted a 'necessary evil' to pursue life goals:

'This to me is a means to an end and I've got to get through this to be where I want'

The notion of control over one's destiny was linked to envisioning a life outside the institution. Patients had frequent thoughts about future plans, which often focused on family:

'I think a lot about the future, because most of my family are aging. And because I'm the oldest man in the family, I need to look after them'

Future plans also reflected a wish to help others:

'I would really like to become a drug counsellor. I think my journey put me in a better position to understand what drug users are going through'

Participants frequently expressed concerns when discussing the future. Some issues related to readjusting to contemporary living:

'Being out will be so strange after so long. The smallest things like crossing the road is going to be a massive step'

Others had concerns around employment:

'I think people wouldn't employ an older person with mental health issues. I can end up relying on benefits, rather than having an active job'

Relapse was also a tangible risk, in the absence of round-the-clock care from the clinical team:

'Because I've done it before, my main concern is: Am I going to fall in the wrong again?'

Given these concerns, several patients exhibited ambivalent feelings about leaving:

'The future scares me a bit. That's why I'm not in a hurry to leave' 
These feelings were prominent among those who risked being moved back to prison, by virtue of their section or because they had a life sentence:

'If I do all the work, they put a referral to medium security. But once I've done all the work in an RSU and I am out, they can lock me back in prison'

\section{Activities}

This theme focussed around participants' feedback around educational/recreational activities and on their suitability for older patients. Participants felt confident that educational activities prepared them to live independently in the community:

'I find IT classes helpful for when I will be outside. It's all done through the internet now'

Patients who regularly attended the local library were also satisfied:

'We got a ward library here, so every Friday we can put our names on the board and go to the library'

When the hospital resources could not fulfil their educational needs, patients had alternative development opportunities:

I've done distance learning through Leicester College on equality and diversity and I'm now nutrition and health'

Leisure activities could also be relaxing and soothing:

'My artwork, it's a chill out for me. It takes me to another place'

Physical activities also provided a social role within the community:

'I got gardening projects starting up. I got plenty to do. I give advice on how to go about in the garden, how to dig holes for plants' 
In addition, such activities promoted outdoor life and contact with nature:

'When I do horticulture, I really feel I'm all one with nature. It is one of the best things I have here'

In relation to the age-friendliness of activities, some patients stated that the activity programme was suitable for any age group:

'It's targeted to all ages. Take the garden for example, anybody can do something there'

Patients with mobility issues, however, complained about the inaccessibility of activities:

'I use a wheelchair or a frame to move and most of the activities are not accessible'

Those patients having stayed in services for a long time reported a loss of enthusiasm in a 'repetitive' programme of activities:

'Because I've been here an unprecedented amount of time, I've done most of the groups and have lost all my enthusiasm'

Not engaging in activities had a negative impact on social inclusion:

'In this place you need to occupy your mind and it's not very helpful if you're sitting there alone with a book on your lap'

For this reason, the few activities specifically for the over 50s received positive feedback:

I attend the Sage group once a month. We go to the OT room and we pick an old film to watch. It's so comfortable and relaxing!'

Therefore, most interviewees advocated for a wider implementation of age-relevant initiatives:

'I'd be happier to see more people together in my age group in social functions or in the gym' 
${ }^{1}$ The Sage group is a monthly social event in Arnold Lodge. It is dedicated to older patients from across all wards, who gather to watch a film.

\section{Social life}

This theme explored how the participants felt they related with members of staff, peers, and external visitors (e.g. family). Interviewees reported that members of staff exhibited genuine commitment to their emotional wellbeing:

'You always have somebody to talk to. They are very caring.'

They were able to respond to their individual needs:

'Some of the patients haven't got the confidence to ask for support, but they recognise that and they sit down with them'

Thus, patients often regarded their clinical team almost as extended family and treasured their support:

I have applied to get leave and the staff have supported that. It's important to know that my clinical team are all behind me'

The patients recognised that attachment was mutual:

'They have a vested interested to get to know you, cos they also spend most of their life here' However, some patients felt that the younger members of staff could be insensitive to agerelated issues:

'Some of them do not understand that people over 50 might have different problems and treat everybody the same'

Participants felt that mental health problems often got in the way of their relationship with peers: 
'They may have issues with their own mental health. And I could have issues too'

Age difference could also impact on good rapport between patients:

'A lot of the times the younger lads don't want you around. And I struggle to have a conversation with them, because most of their things revolves around computers'

Friendships could end when patients are moved across wards of discharged:

'People from the other ward, I spent time with them every day for three years. They knew when I was sad and I knew when I had to cheer them up. So now I am trying to adjust to new people here'

Some patients, especially those on more settled wards, reported being on good terms with their peers:

'I think it's because this is a recovery ward, everybody relates well'

Participants often reported no contact with family:

'I have not been in contact with my daughter for three years'

Barriers to familial relationships included the practicalities of visits:

'My mom is very old and she's had an operation, so she cannot come very often'

Because of the risk for social isolation, befriending schemes were in place:

'A volunteer visitor visits me every month to talk about things that happen outside'

Interviewees wished further implementation of such initiatives:

'I think there should be more opportunities of visits from the outside, so people just don't sit there alone, 


\section{Practical matters}

Participants were asked to reflect on the practical aspects of their daily routines, which might impact on their experience in secure care. Food reviews were overall positive, but several patients reported unmet age-related needs:

'I would appreciate some more food that I can chew. An alternative would be good'

These included feeling uncomfortable to dine in boisterous environments:

'I rarely go to the dining room to eat, because of the noise. You get a lot of chairs scraping on the floor, lots of shouting between patients'

Access to the telephone was another area where the older patients reported age-related unmet needs:

'I can't stand for a long time to be able to use the phone. And sometimes, it can get pretty late before I can make a call, so I just miss out'

The hospital shopping arrangements were found to be age-friendly:

'They give me a shopping sheet, I write down what I want and the porter brings it over in boxes'

Concerns were reported around the availability of funds. For this reason, all the participants described saving strategies:

'I try and save each day some pounds in the safe, in the bank and in the room. Today I spent about $£ 5$ and managed to save $£ 15$ for the next week and that is how I meet my budget'

The interviewees felt that the strict policies around physical contact limited social interaction:

'For me mostly it's just missing on having that interaction with people'. 
There appeared to be some welcome flexibility within the policy that it is allowed to accommodate the needs of partnered patients:

'I have just got married and when my husband visits me, the staff let us hold hands. I really appreciate it'

In relation to sexual expression, the patients generally agreed that intimacy on the ward would be inappropriate and could pose a threat to successful rehabilitation:

'I would view it as inappropriate in a small community, and I think you've got to concentrate on your treatment'

Others reported no sexual needs:

'I have no worries in that sense. You see, I'm older, so these things don't matter to me'

Autoeroticism, however, was still relevant for some, who complained that regulations had become stricter over time:

'We used to receive magazines back then and they helped a lot. We also had a programme called 'Sexcetera' about sexual expression, but they have cancelled it so there's nothing these days'

\section{Recovery}

This theme explores participants' views on recovery and on the different elements that impact on it, including spirituality and treatment. One patient provided an eloquent image of recovery, framed within the metaphor of life as a river:

'Life's like a river. The stream is when you set out for the journey. You are confident, nothing stopping it. Then there's the waterfall. Suddenly the water has no control of where it's going, just falling. You have the disaster and you can't do a thing about it. The fall ends in the lake, 
splashing. That's when recovery begins, when you wake to the fact. You reflect on what caused the fall. Each rock represents an issue that caused the fall and as you sort the rocks out, you let go of the pain from them. That's when you've recovered'

Several elements contributed to success in recovery in the view of participants. Personal commitment was key:

'You're not forced to come here. You need to want to come here and you will eventually get better'

Another relevant factor was support from the community:

'We have group meetings here, so I can come here and say what's on my mind and everybody tries to help me to get around that'

Patients believed that recovery was also linked with quality of life. Although most patients spoke well of certain aspects of treatment, such as the staff and activities, they overall did not think of themselves as having great quality of life:

'It's not life, it's more of an existence. You just get on with your routine, day in and day out'

It was further affected by long stay:

'When you talk about someone being locked up for this amount of time, it's utterly horrendous'

Systemic aspects of secure settings such as restrictiveness, also caused poor quality of life: 'Limitations of what you can do here have a great impact on quality of life. I've always been an outdoor person and now I'm stuck in here'

Recovery was also promoted through a rich spiritual life. A therapeutic element of spirituality was that it provided a sense of peacefulness: 
'Being in the church, makes you feel contended and relaxed. No punishing there'

Spirituality also gave the patients hope for the future:

'It gives me a feeling that I will get free one day'

Religious practices promoted a sense of community:

'You sit with the staff, other patients and the padre...you're all equal in the eyes of God'

Pastoral care was considered akin to talking therapy:

'The chaplain came today. I told him the full story of the psychotic incident and he gave me support'

Given the benefits for the patients, the services were very supportive of any spiritual practice:

'I don't go to church cos it's quite a walk, but I have on ward communion. We also get an Imam, who comes here and a prayer room'

Treatment was another core element of recovery. Very positive feedback was reported around treatment groups:

'We have groups on alcohol, violence, drugs, and relationships. There's always a group for each person's needs'

Participants offered new insight into personal issues:

'You get a different perspective form the staff and from the other peers'

However, the benefits of treatment did not end with group sessions:

I have weekly sessions with my doctor and I can get support from the staff in the evening times. There's always support if you need it' 
In a small number of cases, the interviewees stated that there was little emphasis in secure settings to prepare older patients to move along the care pathway:

'A lot of therapy groups are constructed for younger people. I'm here as an older person, and I have my issues and strengths, which are as important as those of a younger patient'

\section{Physical health}

All the participants experienced physical health conditions. They generally held positive views on how their physical health needs were met:

'We've got a good health care system and if the staff notice anything, they immediately get you sorted'

They frequently noted that their health needs were met better than in the community:

'If I have pain in my back, they'd give me ibuprofen. When I was outside, there were times when I did not have ibuprofen in the medicine box in my flat'

Extra consultations were always available, if needed:

'They do health checking every month. But they come to see you more often if you have got any complaints, injuries or worries'

Information on physical health care was also found to be adequate:

'When I was first diagnosed with diabetes, they immediately gave me information'

Physical activity was a key element to wellbeing, as it boosted self-confidence, helped promoted a sense of purpose and helped obtain visible physical improvements:

'My kids are saying 'Dad, I've never seen you so well'. It shows me that I'm achieving something' 
Gym sessions were especially popular among the older patients:

'All of us over 50 go to the gym. One chap is over 70 and he does all the exercises'

Small fitness suites were also provided on the wards, an initiative which was very well received:

'We have a private gym here on the ward, so, if you feel edgy, you can just go and relax'

The patients, however, reported that much remained to be improved, to ensure equal opportunities to all patients to get active:

'We only get 45 minutes for the gym. And with the time it takes me to get there, I'm looking at 35 minutes'

The gym equipment was reported to be only suitable for younger patients:

'The weights in the gym are too heavy. The over 50s can't lift 30, 40 or 50 kilos'

\section{Service improvement}

We asked participants to report on any possible improvements in the service to enhance their experience. They reported on dedicated units/wards for ageing patients and understaffing issues. It was felt that dedicated units for the over 50s could be beneficial in some respects. They would reduce social isolation:

'People of the same age stick together and you can be a little cut off if you don't relate with the younger people'

They would solve some irreconcilable intergenerational differences:

'I might want to watch something on TV that a very young person wouldn't want to watch'

They would also reduce the potential risk for bullying/victimisation against the older patients: 
'I'd feel a little safer from the young and assaultive guys'

Dedicated wards for the over 50 would be more settled:

'I imagine it'd be a lot quieter. And I can see better conversation, rather than shouting all over the place'

The patients identified barriers to developing these services:

'There's problems with staffing and the prospect of a dedicated ward would be dismissed straightaway'

Other interviewees manifested opposite views toward units for the over 50s:

'I think wards need to be like a community, and in a community, you get people of all ages'

Several interviewees contended mixed environments are more settled:

'When you get younger people coming through, if you have older people around, some of their calm radiates'

They continued that older patients could benefit from mixing with the younger ones:

'The young people can teach us about technology. It's a fast world and you need to be up to date'

Another area in need of improvement was staff-to-patient ratio. Some patients reported that at times wards were run on fewer than three staff. Understaffing could impact on the atmosphere on the ward:

'When the staff do long days, they can get quite edgy, and when you ask for things, they sometimes respond with a nasty follow up'

Another direct consequence was the unequal consideration of patients: 
'People who are high dependent and need attention all the time get it. But the guys who are self-dependent tend to be a little bit forgotten'

Given the staff shortages, movement of personnel across units and wards was frequent. This was cause for further disruption of patients' routine:

'Each ward is different, even the small things, like the boxes where they keep the keys. There should be some sort of uniformity, so members of staff would just need to learn the routine of the patients'

\section{Discussion}

This paper presents findings from a qualitative investigation of a sample of older patients living in secure forensic psychiatric units, providing novel insight into the experience of ageing in secure care.

In line with existing literature on old age forensic psychiatry (Yorston \& Taylor, 2009), our findings highlighted good elements of care, such as physical healthcare provision and educational/cultural opportunities. In addition to the benefits of spirituality documented in the prison literature (Reed, 1980; Bishop et al., 2014; Allen et al., 2013; Allen et al., 2008), our participants also provided positive feedback around support for their spiritual needs.

Our results also highlighted several challenges experienced by the older patients. As found in prison studies (Crawley \& Sparks, 2006; Loeb et al., 2007), participants expressed concerns about the future and their attachment toward forensic units (Yorston \& Taylor, 2009; Doron, 2007; Aday, 1994; Aday \& Webster, 1979), which affected treatment compliance. Long-stay caused dependency from staff, decreased self-confidence to live independently and disenchantment with the benefits of therapy and treatment. Patients' disengagement from participating in the wider community of the hospital was caused by the current programme of 
activities, which was not found to be age-relevant and accessible for those with limited mobility. Accessibility issues have been consistently reported in the forensic literature (Joyce \& Maschi, 2016; Yorston \& Taylor, 2009), showing how little has been done to improve the situation over time.

Regarding the risk of social isolation, in line with previous research (Yorston \& Taylor, 2009), we found that the participants encountered great barriers to building good relationships with their own peers. Although mental health issues were recognised as a mediating factor, poor peer relationships were also the result of intergenerational issues, most patients in mixed-age wards being of younger age. Contrary to previous findings on long-stay patients of all ages (Völlm et al., 2017) and in line with research in old age forensic psychiatry (Yorston \& Taylor, 2009; Crawley, 2005), our participants reported limited contact with their families, a trend which might be related to ageing.

Given the added risk of social isolation, participants frequently discussed potential solutions. In contrast with previous evidence (Yorston \& Taylor, 2009), when asked to comment on possible units for the over 50s, several patients supported mixed-age wards, contending that the benefits of living in a diverse community outnumbered intergenerational issues. Consensus was instead found on the necessity to develop cross-ward social opportunities for older patients, there being just one intiative for over 50s, in the medium secure setting.

In relation to autoeroticism, the participants complained about overly stringent policies. This finding reflected a recent comparison study on policies around sexual expression in European countries, which concluded that the UK adopted the most restrictive regulations (Tiwana, McDonald, \& Völlm, 2016). Quite interestingly, the patients were not as critical in relation to restrictions around sexual relations, contending that they might interfere with treatment. Their stance, however, might also reflect an effort to adapt to the stringent policy. 
This study is characterised by some limitations. Sample size and composition might have affected the generalisability of findings. The only two women and participant of Black ethnicity were from medium security, thus neglecting representation of these populations from other services. In addition, our findings might over represent the views of the more compliant patients, who were more likely to be involved. In addition, the views of patients who lacked capacity to consent were not included.

In relation to data collection, our primarily deductive approach may have imposed themes upon the patients. However, we were aware of this possibility and allowed for new themes to be added, if necessary. Regarding data analysis, qualitative means of investigation require a degree of data interpretation which may alter/filter patients' reports. To mitigate this risk, we kept our text input at a minimum in the results section, to safeguard the integrity of patients' narratives.

In terms of research implications, we were only able to involve three services in our study. Although purposively sampled, our participants might not be representative of the wider population of ageing patients in secure services across the UK and in other countries. We therefore advocate that further research with nationally representative samples be undertaken. Our findings have several implications for policy and practice. Forensic psychiatric services face challenges from the increasing numbers of older patients (Di Lorito, Dening, \& Völlm, 2018). Older forensic patients already experience barriers to recovery, including prolonged stay in secure services, decreased involvement in treatment/activities, and risk of social isolation. This challenges the ability of service providers to grant equal opportunities of care to all ageing individuals, including those in forensic settings, as required by the National Institute for Health and Care Excellence (NICE) guidelines on mental wellbeing and 
independence in ageing people (NICE, 2015) and the Care Act 2015. A timely response is therefore needed.

It remains uncertain whether dedicated units for older patients are practically feasible, financially sustainable and therapeutically effective. However, good practice can be promoted through (further) development of age-relevant approaches. Some examples reported in the literature include Cognitive Stimulation Therapy (CST), reminiscence, speech and language therapy, modifications to environments (e.g. shower chairs, handrails), flexible regimes (e.g. dedicated hours for phone calls) and personalised discharge packages (Natarajan \& Mulvana, 2017).

These strategies need to be tailored to older patients' needs, and so optimal allocation of funding and investment of resources is vital. We have shown that older patients do have views about their treatment and needs and, therefore, systems need to be in place to ensure their active input into how services are organised. Because older patients constitute a numerical minority in secure services, they are often scattered across different wards and they are typically less assertive to voice their needs, they might fail to lobby as effectively as other population groups. Nonetheless, services should actively seek their involvement, so that their distinct service needs can be adequately met. 


\section{References}

Aday, R. H. (1994). Aging in prison: a case-study on new elderly offenders. International Journal of Offender Therapy and Comparative Criminology, 38, 79-91.

Aday, R., \& Webster, E. (1979). Aging in prison: the development of a preliminary model. Offender Rehabilitation, 3, 271-282.

Allen, R. S., Phillips, L. L., Roff, L. L., Cavanaugh, R., \& Day, L. (2008).

Religiousness/spirituality and mental health among ageing male inmates. Gerontologist, 48, 692-697.

Allen, R. S., Harris, G. M., Crowther, M. R., et al. (2013). Does religiousness and spirituality moderate the relations between physical and mental health among aging prisoners?

International Journal of Geriatric Psychiatry, 28, 710-717. https://doi.org/10.1002/gps.3874.

Baldwin, J., \& Leete, J. (2012). Behind bars: the challenges of an ageing population. Australian Journal of Dementia care, 2, 16-19.

Bishop, A. J., Randall, G., Merten, M. J. (2014). Consideration of forgiveness to enhance the health status of older male prisoners confronting spiritual, social, or emotional vulnerability. Journal of Applied Gerontology, 33, 998-1017. https://doi.org/10.1177/ 0733464812456632 .

Braun, V., \& Clarke, V. (2006). Using thematic analysis in psychology. Qualitative Research in Psychology, 3, 77-101.

Coid, J., Fazel, S., \& Kahtan, N. (2002). Elderly patients admitted to secure forensic psychiatry services. The Journal of Forensic Psychiatry, 13, 416-427. DOI: $10.1080 / 09585180210154470$

Cooney, F., \& Braggins, J. (2010). Doing Time. Good practice with older people in prison the views of prison staff. Prison Reform Trust, London.

Crawley E. (2005). Institutional thoughtlessness in prisons and its impacts on the daytoday prison lives of elderly men. Journal of Contemporary Criminal Justice 21, 350-363. https://doi.org/10.1177/1043986205282018.

Crawley, E., \& Sparks, R. (2006). Is there life after imprisonment? How elderly men talk about imprisonment and release. Criminology and Criminal Justice 6, 63-82.

https://doi.org/10.1177/1748895806060667.

Curtice, M., Parker, J., Wismayer, F.S., \& Tomison, A. (2003). The elderly offender: an 11year survey of referrals to a regional forensic psychiatric service. The Journal of Forensic Psychiatry \& Psychology, 14, 253-265. DOI: 10.1080/1478994031000077989

Di Lorito, C., Dening, T., \& Völlm, B. (2018). The characteristics and needs of older forensic psychiatric patients: A UK cross-sectional study. Submitted for publication.

Di Lorito, C., Völlm, B., \& Dening, T. (2018). Psychiatric disorders among older prisoners: a systematic review and comparison study against older people in the community. Aging \& mental health, 22, 1-10. 
Di Lorito, C., Völlm, B., \& Dening, T. (2017). The individual experience of ageing prisoners: systematic review and meta-synthesis through a Good Lives Model framework. International Journal of Geriatric Psychiatry. doi: 10.1002/gps.4762. [Epub ahead of print]

Di Lorito, C., Castelletti, L., Tripi, G., Gandellini, M. G., Dening, T., \& Völlm, B. (2017). The Individual Experience of Aging Patients and the Current Service Provision in the Context of Italian Forensic Psychiatry: A Case Study. Journal of forensic nursing, 13, 118 125.

Doron, I. (2007). Heaven or hell? Aging behind bars in Israel. International Journal of Aging, 9, 145-159. https://doi.org/10.2190/HA.9.2.e.

Drennan, D., Wooldridge, J., Aiyegbusi, A., Alred, D., Ayres, J., Barker, R., Carr, S., Euson, S., Lomas, H., Moore, E., Stanton, D. \& Shepherd, G (2014). Implementing Recovery through Organisational Change Briefing Paper 10. London: Centre for Mental Health and Mental Health Network. Accessed 10.06.2016 at: http://www.imroc.org/wpcontent/uploads/ImROC-briefing-10-Making-Recovery-a-Reality-in-Forensic-Settings-finalfor-web.pdf

Frazer, L. (2003). Ageing inside. school for policy studies working paper series paper number 1. University of Bristol, Bristol. Retrieved from:

http://www.clinks.org/sites/default/files/basic/filesdownloads/Older\%20Prisoners\%20LF\%20 Report\%20sps01_lf.pdf

Joyce, J., \& Maschi, T. (2016). 'In here, time stands still'. The rights, needs and experiences of older people in prison. Irish Penal Reform Trust: Dublin, Ireland.

Lightbody, E., Gow, R.L., \& Gibb, R. (2010). A survey of older adult patients in special secure psychiatric care in Scotland from 1998 to 2007. The Journal of Forensic Psychiatry \& Psychology, 21, 966-974. DOI: 10.1080/14789949.2010.504858

Loeb, S. J., Steffensmeier, D., \& Myco, P. M. (2007). In their own words: older male prisoners' health beliefs and concerns for the future. Geriatric Nursing, 28, 319-329. https://doi.org/10.1016/j.gerinurse.2007.02.001.

Moll, A. (2013). Losing track of time: dementia and the ageing prison population. Mental Health Foundation, London. Accessed RECOOP, 2015.10.17 at:

http://www.mentalhealth.org.uk/content/assets/PDF/publications/losing-track-of-time2013.pdf? view $=$ Standard

Natarajan, M., \& Mulvana, S. (2017). New horizons: forensic mental health services for older people. British Journal of Psychiatry Advances, 23, 44-53.

National Institute for Health and Care Excellence 2015. Mental wellbeing and independence in older people. NICE guideline (NG32). London: National Institute for Health and Care Excellence.

NVivo qualitative data analysis Software (2012). Melbourne, Australia: QSR International Pty Ltd. 
Paradis, C., Broner, N., Maher, L.M., \& O’Rourke, T. (2000). Mentally Ill Elderly Jail Detainees. Journal of Offender Rehabilitation, 31, 77-86. DOI: 10.1300/J076v31n01_05

Reed, M. B. (1980). Aging in a total institution: the case of older prisoners (Doctoral Dissertation). Available at: https://www.ncjrs.gov/pdffiles1/Digitization/ 65232NCJRS.pdf [Accessed 10 Dec 2016]

Resettlement and Care for Older ex-Offenders and Prisoners (RECOOP). (2015). Retrieved from http://www.recoop.org.uk/pages/resources/

Shah, A. (2006). An Audit of a Specialist Old Age Psychiatry Liaison Service to a Medium and a High Secure Forensic Psychiatry Unit. Medicine, Science, and the Law, 46, 99-104.

The Centre for Mental Health (2015). Secure mental health services. Retrieved October 27, 2017, from:

https://www.gov.uk/government/uploads/system/uploads/attachment_data/file/198033/Nation al_Service_Framework_for_Older_People.pdf

Tiwana, R., McDonald, S., \& Völlm, B. (2016). Policies on sexual expression in forensic psychiatric settings in different European countries. International journal of mental health systems, 10,5 .

Tomar, R., Treasaden, I. H., \& Shah, A. K. (2005). Is there a case for a specialist forensic psychiatry service for the elderly? International Journal of Geriatric Psychiatry, 20, 51-56. DOI: $10.1002 / g p s .1247$

Ward, T., Mann, R. E., \& Gannon, T. A. (2007). The Good Lives Model of offender rehabilitation: Clinical implications. Aggression and Violent Behavior, 12, 87-107.

Wong, M.T.H., Fenwick, P.B.C., Lumsden, J., Fenton, G.W., Maisey, M.N., Lewis, P. et al. (1995). Elderly offenders in a maximum security mental hospital. Aggressive Behaviour, 21, 321-324.

Yorston, G.A. (2015). Managing aggression and violence in older people. London: The Royal College of Psychiatrists.

Yorston, G., \& Taylor, P.J. (2009). Older patients in an English high security hospital: a qualitative study of the experiences and attitudes of patients aged 60 and over and their care staff in Broadmoor Hospital. The Journal of Forensic Psychiatry \& Psychology, 20, 255-267. DOI: $10.1080 / 14789940802327259$ 
Figure 1. Selection of participants

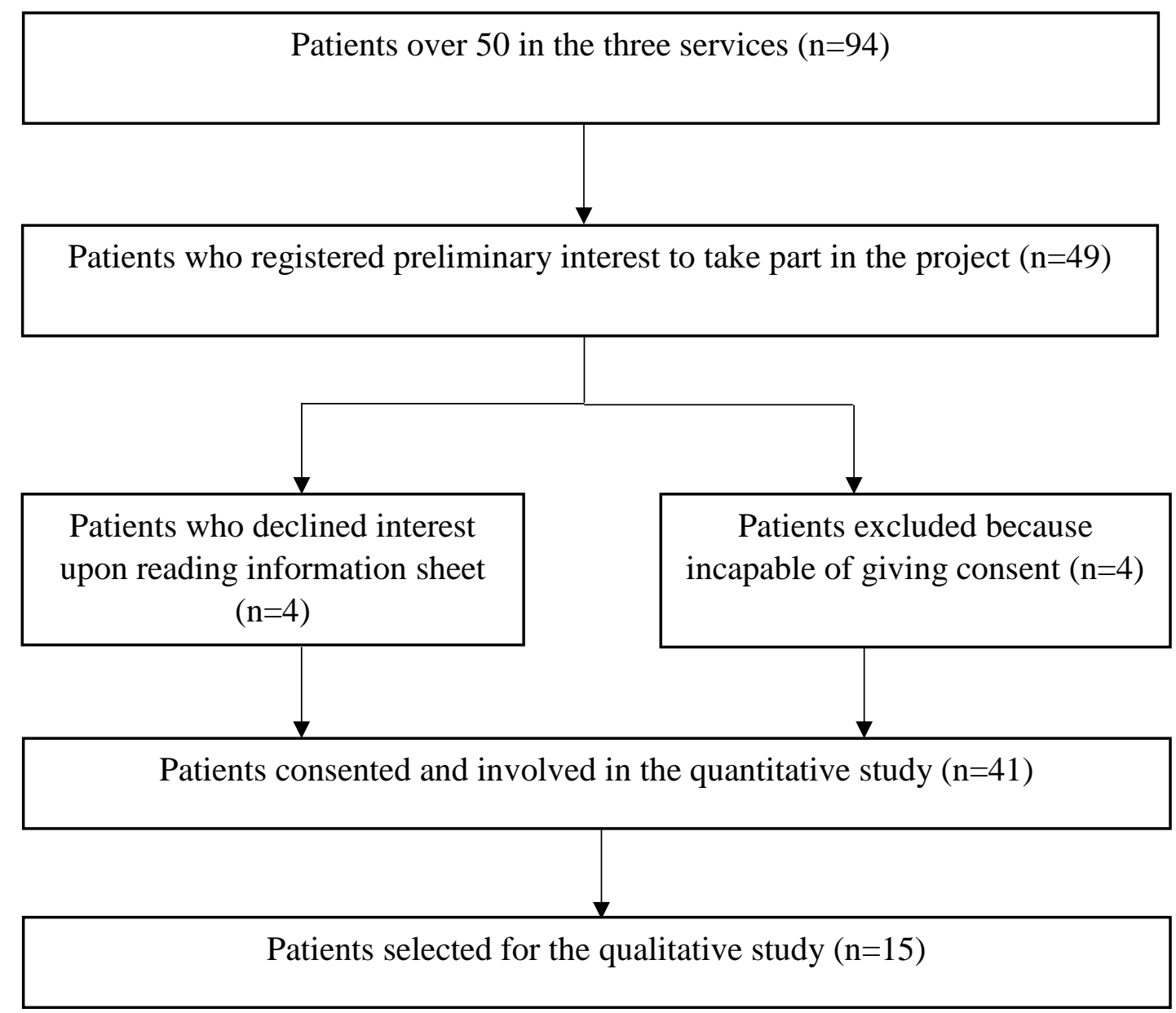


Theme

Self-agency

Activities

Educational/recreational activities

Feedback

Social life

Members of staff

Peers

Family and visitors

Practical matters

Food, dining and telephone

Money and finances

Physical contact

Recovery Views on recovery

Spiritual life

Treatment

Physical health

Physical health care

$$
\text { Shaping one's future }
$$

Explanation

Patient discusses how much they feel in control of their own destiny. Includes perceived

decision-making on treatment-related aspects

Patient discusses any aspect about their future life, including plans, visions, dreams and concerns

Patient describes educational and leisure activities, including hobbies and outdoor activities (e.g.

Gardening and walking)

Patient gives feedback on activities and on age-appropriateness of them. Includes comparisons

with activities in the past and with activities in other settings (e.g. prison)

Patient describes relationship with members of staff, emotional support received, and issues in staff/patient rapport

Patient describes relationship with other patients, emotional support received, mixing issues and bullying/victimisation

Patient describes visitations from family members, and any visitation-related issue. Includes discussion around befriending schemes

Patient discussed food and any issues related to dining. Patient discusses use of telephone and related issues (e.g. privacy, queues, length of calls)

Patient discusses availability of funds and how these are managed. Includes discussion of shopping for goods

Patient gives feedback on policy around physical contact (with patients and visitors). Patient discusses sex life, sex expression and intimate relationships (or lack thereof)

Patient describes views on recovery and the aspects that impact on recovery outcomes

Patient discusses faith/spirituality/religion, how conducive it is to own recovery and how supportive the service is of own religious practice

Patient describes and gives feedback on any type of treatment (e.g. individual/group sessions, psychotropic/psychological therapy)

Patient discusses how physical problems have or have not been taken care of through personal or staff initiatives. Includes feedback on primary physical health care

Physical activity
Patient describes and gives feedback around activities that promote physical health (e.g. gym, walks) 
